\title{
UTILIZAÇÃO DE PEIXES COMO BIOMONITOR PARA FINS DE RADIOPROTEÇÃO AMBIENTAL EM ECOSSISTEMAS AQUÁTICOS CONTINENTAIS. UM MODELO CONCEITUAL
}

\author{
Wagner de Souza Pereira ${ }^{1,2}$, Alphonse Kelecom ${ }^{2,3}$ \& Delcy de Azevedo Py Júnior ${ }^{4}$ \\ ${ }^{1}$ Indústrias Nucleares do Brasil, Caixa postal 961, CEP. 37701-970, Poços de Caldas, Minas Gerais, Brasil. \\ ${ }^{2}$ Programa de pós-graduação em Biologia Marinha, Universidade Federal Fluminense, CP. 100.644, CEP 24001-970, Niterói, RJ, Brasil. \\ ${ }^{3}$ Programa de pós-graduação em Ciência Ambiental, Universidade Federal Fluminense, Avenida Litorânea, S/N, Boa Viagem, Niterói, RJ, Brasil. \\ ${ }^{4}$ Indústrias Nucleares do Brasil, Caixa postal 7, CEP. 46.400-000, Caetité, BA, Brasil. \\ E-mail: wspereira@inb.gov.br
}

\section{RESUMO}

O presente trabalho visa revisar as metodologias de avaliação de dose absorvida em peixes e fornece subsídios para o estabelecimento de uma metodologia de avaliação de impacto ambiental radiológico embasado na avaliação de dose absorvida na biota, com enfoque nos peixes de ambiente aquático continental. Esse enfoque de proteção é recente e incentivado por organismos internacionais e alguns nacionais. Para tanto se fez uma revisão dos métodos de cálculo de dose na biota, passando por suas situações de contorno na elaboração do modelo. Não se fez julgamento de mérito de cada modelo, apenas uma explicitação de seus pressupostos. Baseado na literatura desenvolveu-se um formulário estabelecendo fórmulas de cálculo de fatores de conversão de concentração de atividade em taxa de dose e dos limites derivados para água e sedimento. Desenvolveu-se um modelo de cálculo de taxa de dose absorvida anual, tendo por fundamento o modelo proposto pelo Departamento de Energia (DOE) dos Estados Unidos e proposto um limite de dose baseado no mesmo DOE. Houve a preocupação de se apontar perspectivas de desenvolvimento de avaliações radiológicas ambientais, sendo sugerido uma metodologia de gerenciamento das doses absorvidas pela biota, através dos métodos de valoração do dano ambiental.

Palavras-chave: Radioecologia, radioproteção ambiental, cálculo de dose na biota, dose absorvida, peixe.

\begin{abstract}
UTILIZATION OF FISH AS BIOLOGICAL MONITORS IN CONTINENTAL AQUATIC ECOSYSTEMS FOR ENVIRONMENTAL PROTECTION FROM RADIATION. The present work revisits methodologies to evaluate radiation absorbed dose in fish, while presenting parameters on which a methodology for evaluating environmental radiological impact based on the amount of absorbed dose of the biota can be mounted, herein focusing on continental fishes. This approach is recent and subsided by national and international organisations. A general review of biota dose calculation methods is given. From these data we listed formulas for calculating the conversion factors from activity concentration to dose rate, and to calculate derived water and sediment limits. Herein is also presented a new model to calculate the absorbed dose per year based on the model designed by the US Department of Energy, and a dose limit is proposed based on this model. We tried to point out new perspectives for the development of environmental radiological evaluation methods by suggesting a way of managing the absorbed dose by the biota based on the methods to quantify environmental damage.
\end{abstract}

Keywords: Radioecology, environmental radioprotection, calculation of biota dose, absorbed dose, fish.

\section{INTRODUÇÃO}

A radioproteção humana é uma preocupação recente, que surgiu praticamente junto com o descobrimento dos Raios-X por Roentgem em 1885. Essa preocupação cuida, entre outras coisas, conjunto de medidas que visam proteger o ser humano e seus descendentes contra possíveis efeitos indesejados causados pela radiação ionizante (CNEN 2005). A radioproteção humana está bastante desenvolvida, com padrões rígidos internacionalmente aceitos. Estes padrões são propostos pela International Commission on Radiological Protection - ICRP, sendo acatado por países membros através de legislação própria. 
Já a radioproteção ambiental, preocupação mais recente ainda, tendo sido iniciada na década de 70 do século passado. Cuida do conjunto de medidas que visam proteger a biota dos efeitos indesejados das radiações ionizantes. Diferente da radioproteção humana, a radioproteção ambiental não possui um consenso global (ICRP-60 1991, Pereira \& Py Júnior 2003). No atual estado da radioproteção ambiental, não existem critérios de proteção ambiental explícitos para se evitar os efeitos biológicos indesejados da radiação na biota (ICRP-60 1991, IAEA 2002, Pereira \& Py Júnior 2003).

$\mathrm{Na}$ atualidade a radioproteção ambiental possui vários enfoques, que passam por visões religiosas, legais e culturais, passa ainda por atitudes, que refletem diferentes sistemas subjetivos de proteção ambiental (IAEA 2002). Nenhum desses enfoques estabelece padrões de segurança para a biota, estabelecendo apenas pontos de vistas nos quais programas de radioproteção ambiental devem ser baseados.

A radioproteção ambiental existente atualmente é realizada com a limitação da liberação de efluentes e, baseada em um paradigma proposto pela ICRP no ano de 1977, que afirma "The commission believes that the standard of environmental control needed to protect man to the degree currently thought desirable will ensure that other species are not put at risk..." (ICRP-26 1977).

Este paradigma foi posto em dúvida por vários autores (ICRP-60 1991, IAEA 2002, Amiro 1997, Pentreath \& Woodhead 2001, Pentreath 2002, Woodhead 1979). A própria ICRP reavaliou sua posição e, agora, afirma "Occasionally, individual members of non-human species might be hammed, but not to the extent of endangering hole species or creating imbalance between species" (ICRP-60 1991).

Do controle da concentração de efluentes, passando pelo paradigma humano, a radioproteção ambiental vem evoluindo e a partir da década de 1970, houve várias tentativas de se utilizar o conceito de dose, que é um conceito básico da radioproteção humana. $\mathrm{O}$ conceito de dose, na radioproteção humana, evoluiu de dose absorvida, para dose equivalente dessa, para dose efetiva e, por fim, da dose efetiva para a dose efetiva comprometida. Na radioproteção ambiental, porém, estamos ainda na fase de dose absorvida, caminhando para dose efetiva.
Com o crescimento populacional mundial, houve a necessidade de evoluir do princípio de desenvolvimento desenfreado para desenvolvimento sustentável e de reavaliar e reorganizar o conceito de proteção ambiental e de saúde ambiental, a fim de se fazer frente à crescente demanda por recursos ambientais, mantendo-se a qualidade do meio e o bem estar do Homem.

$\mathrm{O}$ conceito de proteção ambiental ampliou-se nos últimos anos, principalmente após a ECO-92 (United Nations Conference on Environment and Development - UNCED). Essa conferência em sua declaração, a declaração do Rio para o ambiente e desenvolvimento, em seu quarto princípio afirma "environment protection shall constitute an integral part of the development process and cannot be considered in isolation from it" (UN 1992a).

A declaração do Rio contém recomendações específicas sobre rejeitos radioativos e meio ambiente, como se segue:

"Support efforts within IAEA to develop and promulgate radioactive waste safety standards or guidelines and codes of practice as an internationally accepted basis for the safe and environmentally sound management and disposal of radioactive wastes" (UN 1992a).

A Eco-92 nas suas recomendações, foca também a biodiversidade. A Convention on Biological Diversity afirma que a proteção do meio ambiente deve ser feita em termos de preservação da diversidade biológica e da diversidade genética, e de preservação dos recursos biológicos e dos habitats (UN 1992b).

O conceito de desenvolvimento sustentável coloca a proteção ambiental na mesma importância da proteção do homem e afirma que a proteção do ambiente é o primeiro passo para a proteção do homem e, que a mesma precisa ser demonstrada de forma explicita (UN 1992a).

Baseado no conceito de desenvolvimento sustentável (UN 1992a) e no princípio da otimização da radioproteção, (CNEN 2005), o presente trabalho pretende revisar os conhecimentos disponíveis sobre radioproteção ambiental e propor uma metodologia de radioproteção ambiental baseada no conceito de dose absorvida, que foi o princípio da radioproteção humana.

No Brasil existe uma necessidade de avaliação do impacto radiológico ambiental na biota de ecossistemas aquáticos continentais e costeiros. Isso se deve ao fato 
das nossas instalações nucleares estarem próximas a esses sistemas. A Usina nuclear Almirante Álvaro Alberto se encontra no Município de Angra dos Reis e faz liberação controlada de radionclídeos antropogênicos (criados em reatores nucleares) para o ambiente costeiro, o que é uma prática legal. Nossa legislação contempla a proteção da biota de forma indireta, o que não é recomendado pelos organismos internacionais.

As indústrias Nucleares do Brasil, em sua Diretoria de Recursos Minerais, possuem quatro instalações nucleares próximas a ambientes aquáticos continentais. A primeira mina de urânio do Brasil que está em processo de descomissionamento no Município de Caldas, Minas Gerais. Essa mina libera efluentes, após tratamento, para a Represa das Águas Claras que desemboca no Riacho das Antas. Libera, também, para o Córrego da Consulta e para o Córrego do Engenho.

A segunda mina está em fase de operação no Município de Caetité, Bahia. Essa mina se encontra no antigo leito do Córrego da Cachoeira, que foi desviado. Essa instalação possuiu uma barragem, a barragem das águas Claras, que possui peixes introduzidos, que são avaliados sobre o enfoque via de transferência de radionuclideos para o ser humano, é não como impacto na biota.

A terceira instalação é uma jazida, em fase de licenciamento que possui duas represas associadas com peixes locais e introduzidos. A rede hidrográfica da região não é perene, só tendo água em épocas de chuvas, não sustentando a sobrevivência de peixes.

A quarta instalação é uma mina de minerais pesados quepossuem associação com monazita, com impurezas de urânio e tório. Por não existir processamento químico dos minerais não são esperados impactos ambientais relevantes. Ela está associada a ambiente costeiro e de águas continentais.

A necessidade de se conhecer o impacto dos empreendimentos na biota e a não existência de legislação pertinente no Brasil, levou o grupo a estudar as metodologias existentes no mundo e propor uma adaptação para o Brasil

\section{METODOLOGIA PARA CÁLCULO DA DOSE ABSORVIDA - CONCEITOS BÁSICOS}

A necessidade de se estabelecer sistemas que demonstrem de forma inequívoca que a biota está protegida contra efeitos indesejados da radiação ionizante é requisitada por organismos internacionais como (UN 1992a, AEPS 1991, OSPAR 1998, IAEA 1995, todos citados em Brow et al. 2006, ICRP-91 2003, IAEA 2005) e aplicada por vários organismos nacionais. Destes se destacam na Europa os trabalhos da Comissão Européia, com os projetos EPIC, FASSET e ERICA reportados em (EPIC 2003, FASSET 2003, 2004, Beresfort et al. 2003, todos citados em Brow et al. 2006). Na Inglaterra, com a Environment Agency of England and Wales com os trabalhos de Coplestone (Coplestone 2001, 2003, ambos citados em Brow et al. 2006). Na América do Norte, destaca-se o Canadá com o relatório Environment Canada and Health Canada (Environment Canada and Health Canada 2000) e os Estados Unidos da América, com o modelo proposto pelo US Departament of Energy (DOE 2002).

Várias metodologias têm sido estabelecidas para avaliar as doses absorvidas nos organismos, desde modelos simples (Woodhead 1979) até modelos mais complexos. Entre os modelos mais complexos podemos citar os desenvolvidos por Higley e colaboradores (Higley et al. 2003a, Higley et al. 2003b, Higley et al. 2003c). O primeiro trabalho de Higkey e colaboradores (Higley et al. 2003a) estabelece fatores de conversão de concentração de atividade ( $\left.\mathrm{Bq} . \mathrm{Kq}^{-1}\right)$ em taxa de dose absorvida (Gy. $\mathrm{a}^{-1}$ ), já o segundo trabalho, (Higley et al. 2003b) vai um pouco mais longe, estabelecendo um modelo biocinético, baseado nas relações alométricas, dos organismos, a partir do modelo proposto por DOE (2002). Por fim, o último trabalho (Higley et al. 2007), utiliza um modelo probabilistico para estabelecer os fatores de conversão de concentração de atividade em taxa de dose absorvida.

Higley \& Byterk (2007) desenvolveram um modelo baseado no fatores de bioconcentração, outro usando o modelo de caixa (box model) com equações de cinética de primeira ordem para estimar a dinâmica da relação entre meio biótico e abiótico e ainda um modelo baseado na relação alométrica da biota. Esse modelo é, também uma aproximação do modelo proposto por DOE (DOE 2002).

Woodhead (2003) desenvolve outra forma de estimar os efeitos biológicos das radiações ionizantes na biota. Ele parte de modelos populacionais (matriz de população) e de modelos metabólicos para aproximar valores de limites e de efeitos da radiação não no peixe mais na população de peixe. 
Já Brechignac (2003) utiliza a avaliação do risco ecológico (Enviromental Risk Assessment, $E R A$ ) como ferramenta para montar um sistema de proteção à flora e à fauna, visando protegê-los dos efeitos deletérios das radiações ionizantes. Este sistema se torna útil, visto que ele pode ser facilmente assimilado por países que já possuam avaliação de risco ecológico em uso nos seus sistemas de proteção ambiental convencional. O mesmo princípio é aplicado por Fesenko e colaboradores (Fesenko et al. 2006) quando chega a criar um fator de impacto da radiação (Radiatiom Impact Factor, RIF) tanto para o homem como para a biota e compara os dois fatores de impacto estimando qual dos dois grupos (humano ou biota) está mais protegido.

Outros autores trabalham com o princípio ERA. É o caso de Jones e colaboradores (Jones et al. 2003), que além da ERA utilizam modelos dosimétricos analisando as vias criticas de exposição.

Kumlad et al. (2006) focam os fatores de bioacumulação e coeficiente de partição e analisam a importância desses fatores no desenvolvimento de um modelo não-mecanicista.

Kryshev et al. (2007) analisaram a influência das relações ecológicas na resposta a doses absorvidas de radiação, principalmente a relação hospedeiro-parasita e indicam que a existência da relação potencializa o efeito deletério das radiações ionizantes. O modelo usado foi dinâmico.

Brown et al. (2006) focam suas atenções na escolha de organismos alvos da avaliação de dose, se preocupando com o nicho destes organismos, sua sensibilidade intrínseca, e sua sensibilidade radioecológica. $\mathrm{O}$ autor também utiliza metodologia de estimativa de fatores de conversão baseadas na metodologia de Monte Carlo, assim como vários outros autores (IAEA 1979, Copplestone et al. 2001, DOE 2002, Prohl et al. 2003).

Chambers et al. (2006) se preocupam com os fatores de peso da radiação para radiação alfa. A mesma preocupação e demostrada por Amiro (Amiro 1997), só que para todos os tipos de radiação (alfa, beta e gama).

Sazykina \& Kryshev (2003) se preocuparam em montar um banco de dados sobre os efeitos deletérios da radiação ionizante em peixe e com isso estabelecer limites de taxa de dose no modelo por eles proposto. Os efeitos considerados foram morbidez, mortalidade, reprodução, redução da expectativa de vida, incremento do número de anormalidades em embriões, alterações morfológicas e funcionais nas gônadas, esterilidade e efeitos teratogênicos. O modelo de avaliação de dose é bastante similar ao proposto pelo DOE (DOE 2002)

Sazikima (2000), utiliza uma combinação de modelos. Utiliza modelos ecológicos para avaliar as alterações de biomassa do ecossistema, um modelo de distribuição de radionclídeos para avaliar o comportamento dos radionuclídeos nos meios bióticos e abióticos, e por fim um modelo de avaliação de dose, promovendo uma visão integrada desde o termo fonte até a avaliação de dose.

Ulanovsky \& Prohl (2006) desenvolvem uma formulação matemática das possíveis geometrias dos organismos, entre eles dos peixes e, utilizando metodologia baseado em simulação de Monte Carlo aponta as frações de energia absorvida pelos peixes.

O modelo proposto pelo DOE (DOE 2002) é bastante usado na literatura, por ser de fácil utilização e de fácil interpretação dos resultados obtidos. Estas facilidades aliadas ao fato de diversos trabalhos da literatura trabalharem com essa metodologia ou serem baseados nela tem permitido uma comparação mais efetiva dos resultados motivos que fizeram com que o modelo proposto nesse trabalho fosse baseado no modelo apresentado pelo DOE.

Os trabalhos de Pereira e colaboradores são os únicos para o Brasil que utilizam uma metodologia de cálculo de dose absorvida (Pereira et al. 2006a, Pereira et al. 2006b, Pereira et al. 2007a, Pereira et al. 2007b).

\section{METODOLOGIA PARA CÁLCULO DA TAXA DE DOSE ABSORVIDA EM PEIXES}

Esta seção descreve, de uma forma simplificada, os conceitos básicos para o cálculo da taxa de dose absorvida, Limites derivados de concentração e coeficientes de conversão de dose para organismos aquáticos. O modelo abaixo foi adaptado do modelo proposto pelo DOE (DOE 2002).

Os coeficientes de taxa de dose externa (CDE) e interna (CDI) fornecem a taxa de dose externa e interna, respectivamente, por unidade de concentração do radionuclídeo no meio. Para a exposição externa os radionuclídeos emissores de radiação 
penetrante (fótons e elétrons) devem ser considerados, desconsiderando-se as radiações não penetrantes (alfa). Para a exposição interna todas as radiações devem ser consideradas.

A avaliação do impacto ambiental radiológico deve ser feita baseada na dose absorvida total que é a soma das doses externas e internas, que por sua vez é obtida pela multiplicação da taxa de dose pelo tempo de exposição.

Na prática, nem sempre e possível analisar os organismos para avaliar a exposição dos mesmos, isso decorre do fato de nem sempre os organismos estarem disponíveis para a captura, ou não são capturados em quantidade suficiente para a avaliação. Por esta razão desenvolve-se o limite derivado (LD) em outras matrizes. No caso dos peixes, a água e o sedimento. Os LDs são valores de concentração na água ou no sedimento, que baseado em modelos dosimétricos geram o limite de doses absorvidas no organismo em questão. Nessas matrizes contendo concentração de atividades (Bq. ${ }^{-3}$ ou Bq. $\mathrm{kg}^{-1}$ ) menores que o LD os organismos presentes não atingiram o limite estabelecido de dose absorvida.

\section{SITUAÇÃO DE CONTORNO PARA CÁLCULO DO COEFICIENTE DE TAXA DE DOSE EXTERNA}

Para o cálculo do CDE, simplificações do mundo real devem ser realizadas, com compromisso de superestimar os valores para poder assumir uma posição conservadora, que é requerida na radioproteção tanto ocupacional como na ambiental. Para tanto assumimos que:

$1^{\circ}$ ) A fonte (meio ambiente) é assumida como sendo infinita em extensão e contendo concentração uniforme de radionuclídeos.

$2^{\circ}$ ) O organismo é considerado como sendo infinitamente pequeno. A combinação do organismo infinitamente pequeno, com meio homogêneo em concentração de atividade é infinitamente longo, gera uma situação em que o meio emite de forma uniforme e absorve de forma uniforme, sendo o organismo infinitamente pequeno sua dose absorvida será igual à do meio.

$3^{\circ}$ ) Como o organismo é considerado como infinitamente pequeno, todos os fótons e elétrons devem ser considerados, gerando mais uma situação conservadora. $4^{\circ}$ ) O organismo está exposto $100 \%$ do tempo na borda que separa a água do sedimento. Neste caso os compartimentos ambientais são considerados como semi-infinitos e uniformemente contaminados.

\section{CÁLCULO DO COEFICIENTE DE TAXA DE DOSE EXTERNA}

A energia total de todos os fótons e elétrons emitidos no decaimento dos radionuclídeos é expressa como sendo em $\mathrm{MeV}$ por desintegração (Bq. $\mathrm{s}^{-1}$ ). Para a exposição ao meio contaminado a unidade do $\mathrm{CDE}$ será $\mathrm{rad} / \mathrm{d}$ por $\mathrm{pCi} / \mathrm{g}$. A energia emitida em MeV/Bq. $\mathrm{s}^{-1}$ é expressa em termos de unidades mais convenientes para a CDE pela multiplicação de fatores de conversão conhecidos convertendo a energia em $\mathrm{MeV}$ para ergs, energia absorvida em ergs/g para rads, tempo em segundos para tempo em dias e atividade em Bq com atividade em pCi. Logo teremos:

$$
\begin{aligned}
& \left(1 \frac{\mathrm{MeV}}{B q \div \mathrm{s}}\right) \cdot\left(1,6 E-6 \frac{\mathrm{erg}}{\mathrm{MeV}}\right) \cdot\left(0,01 \frac{\mathrm{rad} \div \mathrm{g}}{\mathrm{erg}}\right) . \\
& \left(8,64 E+4 \frac{\mathrm{s}}{\mathrm{d}}\right) \cdot\left(0,037 \frac{\mathrm{Bq}}{\mathrm{pCi}}\right)=5,12 E-5 \frac{\mathrm{rad} / \mathrm{d}}{\mathrm{pCi} / \mathrm{g}}
\end{aligned}
$$

\section{Logo:}

$$
\left(1 \frac{\mathrm{MeV}}{\mathrm{Bq} \div \mathrm{s}}\right)=5,12 E-05 \frac{\mathrm{rad} / \mathrm{d}}{\mathrm{pCi} / \mathrm{g}}
$$

No sistema internacional (SI) a unidade para dose absorvida é o Gray (Gy), para a atividade é o Bequerel (Bq) e de massa é o kg. Como os programas de monitoração ambiental, no Brasil, devem expressar o impacto ambiental em termos de acréscimo de dose por ano, estamos usando a fórmula abaixo para converter as unidades:

$$
\left(1 \frac{\mathrm{MeV}}{B q \div s}\right)=5,04 E-06 \frac{\mathrm{Gy} / \mathrm{a}}{\mathrm{Bq} / \mathrm{kg}}
$$

Para sedimento temos metade desse valor por ele ser um plano semi-infinito, logo temos:

$$
\begin{aligned}
& C D E_{S E D .} \frac{\mathrm{rad} / \mathrm{d}}{p C i / g}=(2,56 E-5) \\
& E_{\text {fótons +elétrons }}\left(\frac{\mathrm{MeV}}{\mathrm{Bq} \div \mathrm{s}}\right)
\end{aligned}
$$


Se utilizarmos os valores em SI, com o tempo em ano temos:

$$
\begin{aligned}
& C D E_{\text {sed }} \frac{G y / a}{B q / k g}=(2,52 E-6) \\
& E_{\text {fótons }+ \text { elétrons }}\left(\frac{M e V}{B q \div S}\right)
\end{aligned}
$$

Para a exposição à água contaminada, assumindo a densidade da água como sendo $1 \mathrm{~g} / \mathrm{cm}^{3}$, temos:

$$
\begin{aligned}
& C D E_{\text {agua }} \frac{\mathrm{rad} / \mathrm{d}}{p C i / l}=(2,56 E-8) \\
& E_{\text {fótons +elétrons }}\left(\frac{\mathrm{MeV}}{B q \div \mathrm{s}}\right)
\end{aligned}
$$

Já utilizando o SI, temos:

$$
\begin{aligned}
& C D E_{\text {ägua }} \frac{G y / a}{B q / m^{3}}=(2,52 E-9) \\
& E_{\text {fótons +elétrons }}\left(\frac{M e V}{B q \div s}\right)
\end{aligned}
$$

SITUAÇÃO DE CONTORNO PARA CÁLCULO DO COEFICIENTE DE TAXA DE DOSE INTERNA

$\left.1^{\circ}\right)$ O organismo é considerado como sendo de tamanho infinito, logo toda a energia emitida pelo radionuclídeo é absorvida pelo organismo.

$2^{\circ}$ ) Os CDI incluem o buildup dos descendentes de meia vida de até 100 anos.

$3^{\circ}$ ) Os radionuclídeos são considerados como distribuídos uniformemente nos tecidos.

\section{CÁLCULO DO COEFICIENTE DE TAXA DE DOSE INTERNA}

Nesta seção apresentamos o cálculo do fator de conversão de dose interna (CDI). O CDI é calculado como a soma de todas as energias de decaimento da energia, multiplicada por fatores de conversão de unidades apropriados. A equação de conversão é mostrada abaixo:

$$
\begin{aligned}
& C D I_{i}=\left(\frac{1 d e \sin * s^{-1}}{B q}\right) \cdot\left(\sum_{i} \sum_{j} Y_{j} E_{j}\right) . \\
& \left(1,6022 E-13 J \mathrm{MeV}^{-1}\right) \\
& \left(3,1536 E 07 s^{*} a^{-1}\right) \frac{1 G y}{1 J^{*} \mathrm{~kg}^{-1}}
\end{aligned}
$$

onde:

$\mathrm{CDI}_{\mathrm{i}}$ (Gy/a por $\mathrm{Bq} / \mathrm{kg}$ de peso úmido de tecido) é o fator de conversão interna que estima a taxa de dose por unidade de concentração para o radionuclídeo i;

$Y_{i}$ é o campo (abundância) de radiação $j$ por desintegração do radionuclídeo $\mathrm{i}$;

$\mathrm{E}_{\mathrm{j}}$ é a energia $(\mathrm{MeV})$ da radiação j por nuclídeo i; e

No caso de se usar as unidades $\mathrm{rad} / \mathrm{d}$ por $\mathrm{pCi} / \mathrm{g}$ a equação acima passa a ser:

$$
\begin{aligned}
& C D I_{i}=\left(\frac{1 d e \sin ^{*} s^{-1}}{B q}\right) \cdot \frac{0,037 B q}{p C i} . \\
& \left(\sum_{i} \sum_{j} Y_{j} E_{j}\right) \\
& \left(1,6022 E-06 e r g * M^{-1}\right) \ldots \\
& \left(8,64 E 04 v^{*} d^{-1}\right)^{0,01 g^{*} r a d} \\
& e r g
\end{aligned}
$$

LIMITES DERIVADOS DA CONCENTRAÇÃO DE RADIONUCLÍDEO NO SEDIMENTO PARA PEIXES EM ECOSSISTEMAS AQUÁTICOS CONTINENTAIS

Este LD é feito para organismos baseado em um modelo conceitual em que o animal vive em um ambiente de interface água-sedimento, com características semi-infinitas. O organismo é considerado infinitamente pequeno e o radionuclídeo uniformemente distribuído no sedimento.

No caso de exposição de peixes a um único radionuclídeo, os LDs são obtidos, nesse método pela equação:

$L D_{s, i, a a}=\frac{365 \cdot L D_{a a}}{F C A_{a a} . C D E_{\text {exterma }, \text { sed }, i}}$

onde:

$\mathrm{LD}_{\mathrm{s}, \mathrm{i}, \mathrm{aa}}(\mathrm{Bq} / \mathrm{kg})$ é a concentração do radionuclídeo i no sedimento que baseada no modelo preliminar gera dose igual ao limite de dose $(0,01 \mathrm{~Gy} / \mathrm{d})$ para animais aquático;

365 (dias por anos) é um fator de conversão;

$\mathrm{CDE}_{\text {externa, sedi, } i}(\mathrm{~Gy} / \mathrm{a}$ por $\mathrm{Bq} / \mathrm{kg})$ é o fator de conversão de dose externa, que é um fator usado para se estimar a taxa de dose absorvida pelos tecidos dos animais aquáticos gerada pelo radionuclídeo I no sedimento; e 
FCA $_{\text {aa }}$ (adimensional) é um fator de correção para área ou tempo de residência do organismo. Esse fator tem como default o valor de 1 .

LIMITES DERIVADOS DA CONCENTRAÇÃO DE RADIONUCLÍDEO NA ÁGUA PARA PEIXES EM ECOSSISTEMAS AQUÁTICOS CONTINENTAIS

$$
L D_{a, i, a a}=\frac{365^{*} L D_{a a}}{F C A_{a a}\left[\begin{array}{l}
\left(0,001^{*} F B A^{*} C D I_{i}\right) \\
+\left(C D E_{\text {agua }, i}\right)
\end{array}\right]}
$$

onde:

$\mathrm{LD}_{\mathrm{a}, \mathrm{iaa}}\left(\mathrm{Bq} \cdot \mathrm{m}^{-3}\right)$ é a concentração no radionuclídeo i na água que, baseada no modelo preliminar gere dose igual ao $\mathrm{LD}_{\text {aа }}\left(0,01 \mathrm{~Gy}\right.$. $\left.\mathrm{d}^{-1}\right)$ para os animais aquáticos;

365 é o fator de correção de dia para ano;

$\operatorname{LD}_{\text {аa }}\left(0,01 \mathrm{~Gy} \cdot \mathrm{d}^{-1}\right)$ é a dose absorvida limite recomendada para organismos aquáticos;

0,001 é um fator de conversão de litro (l) para metro cúbico $\left(\mathrm{m}^{3}\right)$;

FBA (1/kg) é o fator de bioacumulação que consiste na relação entre a concentração por unidade de massa do radionuclídeo i no organismo $(\mathrm{Bq} / \mathrm{kg})$ por concentração por unidade de volume do radionuclídeo i na água $(\mathrm{Bq} / \mathrm{l})$;

$\mathrm{CDI}_{\mathrm{i}}\left(\mathrm{Gy}_{\mathrm{a}} \mathrm{a}^{-1}\right.$ por Bq.kg-1) é o fator de conversão de dose interna usado para estimar a taxa de dose para o tecido do radionuclídeo i;

$\mathrm{CDE}_{\text {agua,i }}\left(\mathrm{Gy}^{-\mathrm{a}^{-1}}\right.$ por Bq.kg $\left.{ }^{-1}\right)$ é o fator de conversão de dose externa que é usado para estimar a taxa de dose do animal aquático em imersão em água contaminada.

\section{MODELO DE AVALIAÇÃO DE DOSE}

AVALIAÇÃO DA DOSE DEVIDO À RADIAÇÃO GAMA:DOSE INTERNA.

Para o cálculo da dose interna devido à radiação gama proveniente do radionuclídeo presente no organismo, temos:

$\mathrm{D}_{\mathrm{Int}, \gamma}=\mathrm{CDI}_{\mathrm{I}} \cdot \mathrm{E}_{\gamma} \cdot \mathrm{N}_{\gamma} \cdot \phi \cdot \mathrm{C}_{\mathrm{O}}$

onde:

$\mathrm{D}_{\text {Int, } \gamma}$ é a dose absorvida interna anual devido à radiação gama $\left(\mathrm{Gy} \cdot \mathrm{a}^{-1}\right)$;

$\mathrm{CDI}_{\mathrm{I}}$ é o fator de conversão de concentração de atividade em taxa de dose anual, devido à radiação gama $\left(\mathrm{Gy} \cdot \mathrm{a}^{-1}\right.$ por $\left.\mathrm{Bq} \cdot \mathrm{Kq}^{-1}\right)$;
$\mathrm{E}_{\gamma}$ é a energia da radiação gama, em MeV;

$\mathrm{N}_{\gamma}$ é a proporção de desintegração que produz radiação gama;

$\phi$ é a fração da energia gama absorvida pelo organismo. Sendo conservativo assumimos que toda a energia é absorvida pelo organismo, logo esse parâmetro é considerado 1, sendo adimensional;

$\mathrm{C}_{\mathrm{O}}$ é a concentração de atividade do radionuclídeo no organismo $\left(\mathrm{Bq} \cdot \mathrm{Kg}^{-1}\right)$.

\section{AVALIAÇÃO DA DOSE DEVIDO À RADIAÇÃO GAMA: DOSE EXTERNA DEVIDO AO RADIONU- CLÍDEO PRESENTE NA ÁGUA}

Para o cálculo da dose externa devido à radiação gama proveniente do radionuclídeo presente na água, temos:

$\mathrm{D}_{\mathrm{Ext}, \gamma, \text { água }}=\mathrm{CDE}_{\text {agua }} \cdot \mathrm{E}_{\gamma} \cdot \mathrm{N}_{\gamma} \cdot(1-\phi) \cdot \mathrm{C}_{\mathrm{A}}$

onde:

$\mathrm{D}_{\text {Ext, } \gamma \text {, água aquáticos é a dose absorvida anual }}$ devido à radiação gama proveniente do radionuclídeo presente na água;

$\mathrm{CDE}_{\text {agua }}$ é o fator de conversão de concentração de atividade em taxa de dose anual, devido à radiação gama $\left(\mathrm{Gy} \cdot \mathrm{a}^{-1}\right.$ por $\left.\mathrm{Bq} \cdot \mathrm{Kq}^{-1}\right)$ emitida pelo radionuclídeo presente na água;

$\mathrm{E}_{\gamma}$ é a energia da radiação gama, em $\mathrm{MeV}$;

$\mathrm{N}_{\gamma}$ é a proporção de desintegração que produz radiação gama;

$\phi$ é a fração da energia gama absorvida pelo organismo emitida pelo radionuclídeo presente na água. Sendo conservativo assumimos que toda a energia é absorvida pelo organismo, logo esse parâmetro é considerado 0 , sendo adimensional;

$\mathrm{C}_{\mathrm{A}}$ é a concentração de atividade do radionuclídeo na água $\left(\mathrm{Bq} \cdot 1^{-1}\right)$.

AVALIAÇÃO DA DOSE DEVIDO À RADIAÇÃO GAMA: DOSE EXTERNA DEVIDO A RADIONUCLIIDEO PRESENTE NO SEDIMENTO

Para o cálculo da dose externa devido à radiação gama proveniente do radionuclídeo presente no sedimento, temos:

$\mathrm{D}_{\text {Ext }, \gamma \text {, sed. }}=\mathrm{CDE}_{\text {sed. }} \cdot \mathrm{E}_{\gamma} \cdot \mathrm{N}_{\gamma} \cdot(1-\phi) \cdot \mathrm{C}_{\mathrm{S}}$

onde: 
$\mathrm{D}_{\text {Ext, } \gamma \text {, sed }}$ é a dose absorvida anual devido à radiação gama proveniente do radionuclídeo presente no sedimento;

$\mathrm{CDE}_{\text {sed. }}$ é o fator de conversão de concentração de atividade em taxa de dose anual, devido à radiação gama $\left(\mathrm{Gy} \cdot \mathrm{a}^{-1}\right.$ por Bq $\left.\cdot \mathrm{Kq}^{-1}\right)$ emitida pelo radionuclídeo presente no sedimento;

$\mathrm{E}_{\gamma}$ é a energia da radiação gama, em MeV;

$\mathrm{N}_{\gamma}$ é a proporção de desintegração que produz radiação gama;

$\phi$ é a fração da energia gama absorvida pelo organismo emitida pelo radionuclídeo presente na água. Sendo conservativo assumimos que toda a energia é absorvida pelo organismo, logo esse parâmetro é considerado 0 , sendo adimensional;

$\mathrm{C}_{\mathrm{S}}$ é a concentração de atividade do radionuclídeo no sedimento $\left(\mathrm{Bq} \cdot 1^{-1}\right)$

\section{DOSE TOTAL DEVIDO À RADIAÇÃO GAMA}

A dose absorvida anual devido à radiação gama é o somatório das doses absorvidas interna, mais externa devido à água e externa devido ao sedimento. Logo temos:

$\mathrm{D}_{\gamma}=\mathrm{D}_{\mathrm{Int}, \gamma+} \mathrm{D}_{\mathrm{Ext}, \gamma, \text { agua }}+\mathrm{D}_{\mathrm{Ext}, \gamma, \text { sed. }}$

AVALIAÇÃO DA DOSE DEVIDA A RADIAÇÃO BETA

AVALIAÇÃO DE DOSE DEVIDO À RADIAÇÃO BETA: DOSE INTERNA

Para o cálculo da dose interna devido a radiação beta proveniente do radionuclídeo presente no organismo, temos:

$\mathrm{D}_{\mathrm{Int}, \beta}=\mathrm{CDI}_{\mathrm{I}} \cdot \mathrm{E}_{\beta} \cdot \mathrm{N}_{\beta} \cdot \phi \cdot \mathrm{C}_{\mathrm{O}}$

onde :

$\mathrm{D}_{\text {Int } \beta}$ é a dose absorvida interna anual devido à radiação beta $\left(\mathrm{Gy} \cdot \mathrm{a}^{-1}\right)$;

$\mathrm{CDI}_{\mathrm{I}}$ é o fator de conversão de concentração de atividade em taxa de dose anual, devido à radiação beta $\left(\mathrm{Gy} \cdot \mathrm{a}^{-1}\right.$ por $\left.\mathrm{Bq} \cdot \mathrm{Kq}^{-1}\right)$;

$\mathrm{E}_{\beta}$ é a energia da radiação beta, em $\mathrm{MeV}$;

$\mathrm{N}_{\beta}$ é a proporção de desintegração que produz radiação beta;

$\phi$ é a fração da energia beta absorvida pelo organismo. Sendo conservativo assumimos que toda a energia é absorvida pelo organismo. Logo esse parâmetro é considerado 1, sendo adimensional;

$\mathrm{C}_{\mathrm{O}}$ é a concentração de atividade do radionuclídeo no organismo $\left(\mathrm{Bq} \cdot \mathrm{Kg}^{-1}\right)$.

AVALIAÇÃO DE DOSE DEVIDO À RADIAÇÃO BETA: DOSE EXTERNA DEVIDO AO RADIONUCLÍDEO PRESENTE NA ÁGUA

Para o cálculo da dose externa devido à radiação beta proveniente do radionuclídeo presente na água, temos:

$\mathrm{D}_{\mathrm{Ext}, \beta, \text { agua }}=\mathrm{CDE}_{\text {agua }} \cdot \mathrm{E}_{\beta} \cdot \mathrm{N}_{\beta} \cdot(1-\phi) \cdot \mathrm{C}_{\mathrm{A}}$

onde:

$\mathrm{D}_{\text {Ext, } \beta \text { água }}$ é a dose absorvida anual devido à radiação beta proveniente do radionuclídeo presente na água;

$\mathrm{CDE}_{\text {agua }}$ é o fator de conversão de concentração de atividade em taxa de dose anual, devido à radiação beta $\left(\mathrm{Gy} \cdot \mathrm{a}^{-1}\right.$ por Bq $\left.\cdot \mathrm{Kq}^{-1}\right)$ emitida pelo radionuclídeo presente na água;

$\mathrm{E}_{\gamma}$ é a energia da radiação beta, em $\mathrm{MeV}$;

$\mathrm{N}_{\gamma}$ é a proporção de desintegração que produz radiação beta;

$\phi$ é a fração da energia beta absorvida pelo organismo emitida pelo radionuclídeo presente na água. Sendo conservativo assumimos que toda a energia é absorvida pelo organanismo. Logo esse parâmetro é considerado 0 , sendo adimensional;

$\mathrm{C}_{\mathrm{A}}$ é a concentração de atividade do radionuclídeo na água $\left(\mathrm{Bq} \cdot 1^{-1}\right)$.

AVALIAÇÃO DE DOSE DEVIDO À RADIAÇÃO BETA: DOSE EXTERNA DEVIDO AO RADIONUCLÍDEO PRESENTE NO SEDIMENTO

Para o cálculo da dose externa devido à radiação gama proveniente do radionuclídeo presente no sedimento, temos:

$\mathrm{D}_{\text {Ext, } \beta \text {, sed. }}=\mathrm{CDE}_{\text {sed. }} \cdot \mathrm{E}_{\beta} \cdot \mathrm{N}_{\beta} \cdot(1-\phi) \cdot \mathrm{C}_{\mathrm{S}}$

onde:

$\mathrm{D}_{\text {Ext, } \beta \text {, sed. }}$ é a dose absorvida anual devido à radiação beta proveniente do radionuclídeo presente na sedimento; 
$\mathrm{CDE}_{\text {sed. }}$ é o fator de conversão de concentração de atividade em taxa de dose anual, devido à radiação beta $\left(\mathrm{Gy} \cdot \mathrm{a}^{-1}\right.$ por Bq . $\left.\mathrm{Kq}^{-1}\right)$ emitida pelo radionuclídeo presente no sedimento;

$\mathrm{E}_{\beta}$ é a energia da radiação beta, em $\mathrm{MeV}$;

$\mathrm{N}_{\beta}$ é a proporção de desintegração que produz radiação beta;

$\phi$ é a fração da energia beta absorvida pelo organismo emitida pelo radionuclídeo presente na água. Sendo conservativo assumimos que toda a energia é absorvida pelo organismo, logo esse parâmetro é considerado 0 , sendo adimensional;

$\mathrm{C}_{\mathrm{S}}$ é a concentração de atividade do radionuclídeo no sedimento $\left(\mathrm{Bq} \cdot \mathrm{l}^{-1}\right)$.

\section{DOSE TOTAL DEVIDO À RADIAÇÃO BETA}

A dose absorvida anual devido à radiação beta é o somatório das doses absorvidas interna, mais externa devido à água e externa devido ao sedimento. Logo temos:

$\mathrm{D}_{\beta}=\mathrm{D}_{\mathrm{Int}, \beta+} \mathrm{D}_{\mathrm{Ext}, \beta \text {, água }}+\mathrm{D}_{\text {Ext, } \beta \text {, sed. }}$

\section{AVALIAÇÃO DA DOSE DEVIDA À RADIAÇÃO} ALFA

\section{AVALIAÇÃO DE DOSE DEVIDO À RADIAÇÃO ALFA: DOSE INTERNA}

Para o cálculo da dose interna devido à radiação alfa proveniente do radionuclídeo presente no organismo, temos:

$\mathrm{D}_{\text {Int, } \alpha}=\mathrm{CDI}_{\mathrm{I}} \cdot \mathrm{E}_{\alpha} \cdot \mathrm{N}_{\alpha} \cdot \phi \cdot \mathrm{C}_{\mathrm{O}}$

onde :

$\mathrm{D}_{\text {Int, } \alpha}$ é a dose absorvida interna anual devido à radiação alfa $\left(\mathrm{Gy} \cdot \mathrm{a}^{-1}\right)$;

$\mathrm{CDI}_{\text {I }}$ é o fator de conversão de concentração de atividade em taxa de dose anual, devido à radiação alfa $\left(\mathrm{Gy} \cdot \mathrm{a}^{-1}\right.$ por Bq $\left.\cdot \mathrm{Kq}^{-1}\right)$;

$\mathrm{E}_{\alpha}$ é a energia da radiação alfa, em MeV;

$\mathrm{N}_{\alpha}$ é a proporção de desintegração que produz radiação alfa;

$\phi$ é a fração da energia alfa absorvida pelo organismo. Sendo conservativo assumimos que toda a energia é absorvida pelo organismo. Logo esse parâmetro é considerado 1 , sendo adimensional;

$\mathrm{C}_{\mathrm{O}}$ é a concentração de atividade do radionuclídeo no organismo $\left(\mathrm{Bq} \cdot \mathrm{Kg}^{-1}\right)$.

\section{AVALIAÇÃO DE DOSE DEVIDO À RADIAÇÃO ALFA: DOSE EXTERNA}

A radiação alfa por não possuir poder de penetração é desconsiderada na avaliação de dose externa.

\section{DOSE TOTAL DEVIDO À RADIAÇÃO ALFA}

A dose absorvida anual devido à radiação alfa e igual à dose absorvida interna.

$\mathrm{D}_{\alpha}=\mathrm{D}_{\text {Int }, \alpha}$

\section{DOSE TOTAL NO ORGANISMO}

A dose absorvida total devido ao radionuclídeo presente no organismo, água e sedimento, é o somatório da dose devido à radiação gama, mais beta e mais alfa, sendo o somatório das equações (20), (24) e (26). Logo, temos:

$\mathrm{D}_{\mathrm{T}}=\mathrm{D}_{\gamma}+\mathrm{D}_{\beta}+\mathrm{D}_{\alpha}$

\section{CRITÉRIOS DE DECISÃO}

$\mathrm{Na}$ avaliação do impacto ambiental radiológico é necessário estabelecer critérios de decisão para o se explicitar as boas práticas de radioproteção ambiental, garantindo que a biota está devidamente protegida. O primeiro enfoque é se estabelecer limites de dose absorvida na biota que não devam ser ultrapassados.

No presente trabalho propomos a utilização do limite baseado no estabelecido pelo DOE, que é de $10 \mathrm{mGy} \cdot \mathrm{d}^{-1}$, amplamente aceito na literatura consultada (EPIC 2003, FASSET 2003, 2004, Beresfort et al. 2003, Higley et al. 2003a, Higley et al. 2003b, Higley et al. 2003c). Como no Brasil a radioproteção é feita em termos anuais, temos que o limite em termos anuais corresponde a 3,65Gy. $\mathrm{a}^{-1}$.

\section{PERSPECTIVAS}

Atualmente tem-se dado grande enfoque nos modelos dosimétricos determinísticos (EPIC 2003, FASSET 2003, ambos citados em Brow et al. 2003, Higley et al. 2003a). Começou-se a desenvolver modelos com composição de funções alométricas (DOE 2002). Modelos probabilísticos estão sendo 
desenvolvidos (Higley et al. 2003c). A abordagem de avaliação de risco ecológico também tem sido desenvolvida (Brenignac 2003). Todos eles no cálculo da dose e sua comparação com um limite, geralmente o proposto pelo DOE (DOE 2002).

Uma abordagem bastante recente consiste na utilização da valoração do dano ambiental como ferramenta de decisão na radioproteção ambiental (Pereira et al. 2004, 2006). Esta nova ferramenta permite estabelecer faixas de doses absorvidas e assume que o meio ambiente deva ser ressarcido com obras de melhorias, com graduação por faixa de impacto e ainda o estabelecimento de um limite máximo que não deverá ser ultrapassado. Este princípio é chamado de poluidor-pagador, e tem sido utilizado como ferramenta importante para a proteção ambiental (IAEA 2002). Pereira et al. (2006a), aponta uma metodologia de valoração baseada na função dose-resposta, como a mais indicada para ser utilizada na valoração do dano ambiental causado pela radiação ionizante. Este princípio, função dose-resposta, não é por si só um mecanismo de valoração do dano ambiental, mas permite estabelecer uma relação usada como input para o processo de valoração.

Como perspectivas de médio prazo o desenvolvimento de novos modelos, baseados nas funções alométricas, com enfoque probabilístico servindo de input para processos de valoração ambiental parece ser o futuro da radioproteção ambiental.

Cabe ressaltar que na radioproteção humana, nenhum benefício concedido ao trabalhador pode justificar a exposição desnecessária. Esse fundamento de radioproteção parece ir de encontro com o princípio poluidor-pagador. Esse enfoque deve ser considerado com cuidado, pois a exposição do meio ambiente também deve ser justificada. O que se propõe é uma compensação por possíveis efeitos deletérios na biota. Tal custo ambiental deve ser dividido com a sociedade e o causador do efeito deve ser penalizado. Essa metodologia permite incentivar o princípio de radioproteção conhecido como otimização (CNEN 2005). Esse princípio prega que os procedimentos devem ser evitados tentando minimizar as doses ao qual os trabalhadores estão expostos. Essa mesma filosofia deve ser implantada para o meio ambiente e as punições devem ser diretamente proporcional ao potencial do dano ambiental valorado.

\section{REFERÊNCIAS}

AMIRO, B.D. 1997. Radiological dose conversion factor for generic non-human biota used for screening potential ecological impacts. Journal of Environment Radioactivity, 35(1): 35-51.

BRECHIGNAC, F. 2003. Protection of the environment: how to position radioprotection in an ecological risk assessment perspective. The Science of the Total Environment, 307: 35-54. BROWN, J.E.; HOSSEINI, A.; BØRRETZEN, P \& THØRRING, H. 2006. Development of a methodology for assessing the environmental impact of radioactivity in Northern Marine environments. Marine pollution Bulletin, 52: 1127-1137.

CHAMBERS, D.B., OSBORNE, R.V. \& GARVA, A.L. 2006. Choosing an alpha radiation weighting factor for doses to nonhuman biota. Journal of Environmental Radioactivity. 87: 1-14.

CNEN, CNEN NN-3.01. 2006. Diretrizes básicas de proteção radiológica. Rio de Janeiro, 26p.

DOE (DEPARTMENT OF ENERGY). 2002. A graded approach for evaluating radiation dose to aquatic and terrestrial biota. Doe-std-1153-2002 Module 3 Methodos derivation, 58 pp,

ENVIRONMENT AND HEALTH CANADA. 2000. Releases of radionuclides fron nuclear facilites. Impacto n non-human biota. Priority substances list assessment repor. Canadian environmet protection act 1999. Evironment Canada. Ottawa. 98p.

FESENKO, S.V.; ALEXAKHIN, R.M.; GERAS'KIN, S.A.; SANZHAROVA, N.I.; SPIRIN, Y.V.; SPIRIDONOV, S.I.; GONTARENKO, I.A. \& STRAND, P. 2006. Comparative radiation impact on biota and man in the area affected by the accident at the Chernobyl nuclear power plant. Journal of Environmental Radioactivity 80: 1-25.

HIGLEY, K.A.; DOMOTOR, S.L.;ANTONIO, E.J.; \& KROCHER, D.C. 2003a. A derivation of a screening methodology for evaluating radiation dose to aquatic and terrestrial biota. Journal of Environmental Radioactivity, 66: 41-59.

HIGLEY, K.A.; DOMOTOR, S.L.; \& ANTONIO, E.J. 2003b. A kinetic-allometric approach to predidting tissue radionuclide concentrations for biota. Journal of Environmental Radioactivity, 66: 61-74.

HIGLEY, K.A.; DOMOTOR, S.L. \& ANTONIO, E.J. 2003c. A probabilistic approach to obtaining limiting estimates of radionuclide concentration in biota. Journal of Environmental Radioactivity, 66: 75-87.

HIGLEY, K.A. \& BYTWERK, D.P. 2007. Generic approaches to transfer A kinetic-allometric approach to predidting tissue radionuclide concentrations for biota. Journal of Environmental Radioactivity, 99: 4-23.

IAEA (INTERNATIONAL ATOMIC ENERGY AGENCY). 1979. Methodology for assessing impacts of radioactivity in aquatic ecosystems. Technical Report Series No. 190, Vienna. 
IAEA (INTERNATIONAL ATOMIC ENERGY AGENCY). 2002. Ethical considerations in protecting the environment from the effects of ionizing radiation. A report for discussion. IAEA TECDOC-1270, Austria.

IAEA (INTERNATIONAL ATOMIC ENERGY AGENCY). 2005. Conf. on protection of the environment fron de effects of ionising radiation 6-10 October. 2003, stockholm, Sweden. IAEA cn109/37.9, 2005

ICRP 60. 1991. Recommendations of the International Commission on Radiological Protection. ICRP Publication 60, Pergamon press, oxford and New York (1991).

ICRP 26. 1977. Recommendations of the International Commission on Radiological Protection. ICRP Publication 26, Pergamon press, oxford and New York (1977).

ICRP-91. 2003. A framework for assessing the impact of ionising radiation on non-human species. Pergamon press, oxford and New York (2003).

JONES, D.; DOMOTOR, S.; HIGLEY, K.A.; KOCHER, D \& BILYARD, G. 2003. Principles and issues in radiological ecological risk assessment. Journal of environmental radioactivity. 66: 19-39.

KRYSHEV, A.I.; SAZYKIA, T.G.; \& SANINA, K.D. 2007. Modelling of effects due to chroic exposure of a fish population to ionizing radiation. Radiation and Environmental Biophysics. 46: 123-135.

KUMBLAD, L.; KAUTSKY, U. \& NAESLUND, B. 2007. Transport and fate of raionucldes in aquatic environments - The use of ecosystem modeling for exposure assessment of nuclear facilites. Journal of Environmental Radioactivity. 87: 107-129.

PENTREATH, R.J. 2002. Radiation protection of people and environment developing a common approach. Journal of Radiological Protection, 22: 1-12.

PENTREATH, R.J. \& WOODHEAD, D.S. 2001. A systems or protecting the environment from ionizing radiation: selecting reference fauna and flora, and possible dose models and environmental geometrics that could be applied to them. Science of the Total Environment. 277: 33-43.

PENTREATH, R.J. 1999. A system for radiological protection on the environment: some initial thoughts and Ideas. Journal of Radiological Protection. 19(2) 117-128,

PEREIRA, W.S. \& PY JÚNIOR, D.A. 2003. Environmental danage valuation as radiation protection tool. Pp. 1-6. In: International conference on the protection of the environment fron effects of ionizing radiation. Stockholm.

PEREIRA, W.S.; PY JÚNIOR, D.A. \& PIRES, F.C.A.C. 2004. Utilização da valoração do dano ambiental como ferramenta de radioproteção ambiental. In: Congresso da Sociedade Brasileira de Biociências Nucleares, Fortaleza, Brasil
PEREIRA, W.S.; PY JÚNIOR, D.A. \& KELECOM, A. 2006a. Environmental damage valuation as radiation protection tool. . In: Congresso Brasileiro de Energia, Rio de Janeiro, Brasil PEREIRA, WS; KELECOM, A. \& PY JÚNIOR, D.A. 2006b. Proposta de um programa de proteção ambiental do meio ambiente marinho, contra radionuclídeos, baseado no cálculo de dose absorvida. In: Primeiro Congresso Brasileiro de Biologia Marinha, UFF, Niterói, Brasil

PEREIRA, W.S.; KELECOM, A.; GOUVEA, R.C.S. \& PY JÚNIOR, D.A. 2006c. O cálculo da dose absorvida em peixes como parâmetro de radioproteção no ambiente marinho. In: Primer congresso americano da IRPA, de 3 a 8 de Setembro de 2006, Acapulco, México.

PEREIRA, W.S.; KELECOM, A. \& PY JÚNIOR, D.A. 2007a. Absorbed Dose Rate Due to Intake of Natural Radionuclides by Cará Fish (Geophagus brasiliensis, Quoy \& Gaimard, 1824) Estimated near the Uranium Minning at Caldas, Minas Gerais, Brazil.

PEREIRA, W.S.; KELECOM, A. \& PY JÚNIOR, D.A. 2007b. Absorbed Dose Rate Due to Intake of Natural Radionuclides by Tilapia Fish (Tilapia nilotica, Linnaeus, 1758) Estimated Near Uranium Anomaly at Santa Quitéria, Ceará, Brazil.

SAZYKINA, T.G. 2000. ECOMOD An ecological approach to radioecological modelling. Journal of Environmental Radioactivity. 50: 207-220.

SAZYKINA, T.G. \& KRYSHEV, A.I. 2003. EPIC database on the effects of chronic radiation in fish: Russian/FSU data. Journal of Environmental Radioactivity, 68: 65-87.

STRAND, P. \& OUGHTON, D (eds). 2002. Protection of the environment: current status and future work. IUR report 3, ISBN 82-90362-18-8, 23pp. (disponível em www.iur-uir.org).

ULANOVSKY, A \& PROHL, G. 2006. A pratical method for assessment of dose conversion coefficients for aquatic biota. Radiation and Environmental Biophysics, 45: 203-214.

United Nations. 1992a. United Nations Conference on environment and development, Rio declaration on environment and Development.

United Nations. 1992b. United Nations Conference on environment and development, Convention on biological diversity.

WOODHEAD, D.S. 1979. Methods of dosimetry for aquatic organisms, in methodology for assessing impacts of radioactivity in aquatic ecosystems, IAEA, Tech. Report, 190: 43-96.

WOODHEAD, D.S. 2003. A possible approach for the assessment radiation effects on populations of wild organisms in radionuclide-contaminated environments? Journal of Environmental Radioactivity, 66: 181-213.

Submetido em 15/03/2008. Aceito em 20/09/2008. 